\title{
Analysis of Competition Fronting the Popularity of Content in Social Networks
}

\author{
Siham Hafidi, Mohamed Baslam*, Rachid El Ayachi \\ Morocco Sultan Moulay Slimane University, TIAD Laboratory, Beni Mellal. Morocco
}

\begin{tabular}{l}
\hline \hline Article Info \\
\hline Article history: \\
Received Feb 13, 2017 \\
Revised Jun 24, 2017 \\
Accepted Jul 15, 2017 \\
\hline
\end{tabular}

\section{Keywords:}

Content Provider

Game theory

Popularity

Social Networks

Visibility

\begin{abstract}
In recent years, on the Internet, there is a real phenomenon: the development of social networks that are becoming more popular and more used. Social networks have millions of users worldwide. This provides an opportunity for companies to reach out a large and diverse audience for their advertising campaigns. They do this by creating and spreading content across the social network, which will increase the chance of visibility for their contents, which for them is the assurance of being popular. Every content requires time to reach a specific destination on the networks. In this article, we study competition between several contents that look for attracting more consultations, each characterized by some given popularity. There is competition between the contents of a limited set of destinations. We first model our system, we then study the competition between contents by using the game theory to analyze this behavior. We finally provide numerical results, which provide insights into the effect of various parameters of the system.
\end{abstract}

Copyright @ 2017 Institute of Advanced Engineering and Science. All rights reserved.

\section{Corresponding Author:}

Mohamed Baslam,

Department of Computer Science, Faculty of Science and Techniques

University of Sultan Moulay Slimane

Faculté des Sciences et Techniques, B.P: 523 Béni-Mellal Morroco

Email: baslam.med@gmail.com

\section{INTRODUCTION}

In recent years, the Internet and the Web in particular have undergone profound changes, and there is a phenomenon that appeared with the Web development including: social networks. The emergence of social networks is related to technological and technical revolutions. These revolutions have allowed faster interactions with web pages. Therefore, the number of members of these social networks has prolonged, because the interactions have become more faster, and the consultation of the Internet has become more comfortable.

A social network can be defined as a set of relationships between a set of actors. Intuitively, network is a strategic game, for the reason that a network is a group of people connected to each other and which are in interaction with other people, and they wait, usually a gain as a result of their interactions. To reach the personal goal, sometimes a player acts selfishly toward other people and sometimes it agrees with them.

Initially, Social networks are developed to provide communication and data sharing between users, but they have also become a new means of promotion for companies. Therefore, this article focuses on the study of a competitive situation between content who looking to attract more consultations and maximize their own profits.

The competition is formulated as a non-cooperative game, where each content is characterized by a price and a cost of production. 
In this work, we present a competitive situation among the contents of a social network, or each content seeks to attract more customers and maximize its own revenues, while unveiling the modeling of the problem, formulation and analysis of the game.

\section{Related Works:}

Applying game theory in competition problems in social networks is an active research area, in which game-theoretic models have been developed and studied in the last decades, [1-3].

Past works in this area have focused on the competition between contents in social networks over popularity and over visibility space, in conjunction with advertisement issues to promote content.

Both fully dynamic [3] models as well as semi-dynamic [1] models have been proposed. It has been noted that these problems are similar in nature to the problem of competition over shelf space.

Our model is inspired from [1], where Altman handled a situation of competition between service providers that create content, and that use methods of acceleration which increases the popularity of their content and in particular the publication in social networks.

Altman examined $x_{i}(\mathrm{t})$ as the number of destinations that have obtained by time $\mathrm{t}$ content from seed $i$, and $x(t)=\sum_{i=1}^{N} x_{i}(\mathrm{t})$, and he showed that:

$$
\lim _{t \rightarrow \infty} x_{i}(\mathrm{t})=\mathrm{M} \frac{\lambda_{i}}{\lambda}
$$

With $\lambda=\sum_{i=1}^{N} \lambda_{i}$

\section{UTILITY MODEL}

In this section, we formulate the interaction among contents as a non-cooperative game. We assume that there is a set $\mathrm{N}$ of $\mathrm{N}$ competing contents of a social network, and let $\mathrm{M}$ the number of subscribers for access to the network and who are interested in the $\mathrm{N}$ content.

We assume that opportunities for accessing a content $\mathrm{n}$ arrive at destination $\mathrm{m}$ according to an exponential law with parameter starting at time $\mathrm{t}=0$.

We assume that the possibilities of access to content $\mathrm{i}$ arrive at destination $\mathrm{m}$ according to an exponential law parameter that maintains a time. The time for a content $\mathrm{i}$ arrived at a destination $\mathrm{m}$ is exponentially distributed with the parameter, and according to the mean or expected value of an exponentially distributed random variable $t$, the average time for a content $\mathrm{i}$ arrived at a destination $\mathrm{m}$ is exactly the opposite of parameter and we note:

\section{$=1 /$.}

We shall consider opportunities of acceleration rates of content by putting some efforts, such as the publication of these contents in different social networks. Without any such effort, we assume that=whereare some constants.

Thus, we assume that each content $\mathrm{i}$ has a (unique) utility function. This function depends on the content variable, but also on cost and price.

The utility function of the content $\mathrm{i}$ is given by the following formula:

$$
\mathrm{U}_{\mathrm{i}}\left(\lambda, \mathrm{p}_{i}, \gamma_{i}\right)=\mathrm{p}_{i} \times \mathrm{M} \frac{\lambda_{\mathrm{i}}}{\sum_{\mathrm{j}=1}^{\mathrm{N}} \lambda_{\mathrm{j}}}-\gamma_{i} \mathrm{M}\left(\lambda_{\mathrm{i}}-\phi_{\mathrm{i}}\right)
$$

Where $\mathrm{p}_{i}$ and $\gamma_{i}$ are two parameters, which respectively represent the price and the cost of production of a content. This function refers to net incomes of content, and which is represented by the difference between total income and expenses of this content.

This function refers to net incomes of content, and which is represented by the difference between total income and expenses of this content.

Where:

and:

$$
\mathrm{p}_{i} \times \mathrm{M} \frac{\lambda_{\mathrm{i}}}{\sum_{\mathrm{j}=1}^{\mathrm{N}} \lambda_{\mathrm{j}}}: \text { the total income, } \gamma_{\mathrm{i}} \mathrm{M}\left(\lambda_{\mathrm{i}}-\phi_{\mathrm{i}}\right): \text { the total cost to produce the content. }
$$

$\gamma_{i}:$ production cost of the content $I$,

$\mathrm{p}_{i}$ : the price of content,

$\phi_{i}$ : popularity rate of content $i$,

M: the total number of subscribers to the network.

\section{A NON COOPERATIVE GAME FORMULATION}


For a precise formulation of a non-cooperative game, we have to specify the number of players, the possible actions available to each player, and any constraints that may be imposed on them, and finally the utility function of each player which she attempts to optimize.

Here we will consider the following formulation of games:

Let $J=\left\{N, U_{-} i\left(\lambda, p \_i, \gamma \_i\right), \lambda=\left(\lambda \_1, \ldots, \lambda \_N\right)\right\}$ denote the non-cooperative $\lambda$ game, where:

$\mathrm{N}=\{1 \ldots \mathrm{N}\}$ is the index set identifying the contents,

$\mathrm{U} \_\mathrm{i}$ is the utility function,

$\lambda=\left(\lambda \_1 \ldots \lambda \_N\right)$ is the strategy space of each content,

$\mathrm{P}=\left(\mathrm{p} \_-\overline{1} \ldots \mathrm{p} \_\overline{\mathrm{n}}\right)$ price vector,

$\gamma=\left(\gamma_{-} 1 \ldots \gamma_{-} \mathrm{N}\right)$ cost vector.

We assume that each content $i$ has only one endpoint that is $\lambda \_i$, and seeks to maximize his utility function. $\lambda \_i$ represents an equilibrium for each content $i$, when $\lambda \_i$ maximizes $U_{-} i\left(\lambda, p \_i, \gamma \_i\right)$, we can express that as:

$$
\mathrm{U}_{\mathrm{i}}\left(\lambda^{*}, \mathrm{p}_{\mathrm{i}}, \gamma_{\mathrm{i}}\right)=\max _{\lambda} \mathrm{U}_{\mathrm{i}}\left(\lambda_{1}, \ldots, \lambda_{\mathrm{N}}, \mathrm{p}_{\mathrm{i}}, \gamma_{\mathrm{i}}\right)
$$

\subsection{The Nash Equilibrium}

The Nash equilibrium is the natural concept solution of non-cooperative games, which describes a state of a game where no player has sensitive to deviate unilaterally from its current strategy. In this section, we first will study the Nash equilibrium solution for the induced game, we will show that a Nash equilibrium solution exists and is unique by using the theory of concave games [4].

We recall that a non-cooperative game $\mathrm{J}$ is called concave if all player's utility functions are strictly concave with respect to their corresponding strategies [4]. According to [4], a Nash equilibrium exists in a concave game if the strategy space is compact and convex, and the utility function that any given player seeks to maximize is concave in its own strategy and continuous at every point in the product strategy space.

The Nash equilibrium in $\lambda$ is formally defined as:

Definition1: The vector $\lambda^{*}=\left(\lambda_{1}{ }^{*}, \ldots, \lambda_{N}{ }^{*}\right)$ is a Nash equilibrium of the game $\mathrm{J}=\left\{\mathrm{N}, \mathrm{U}_{-} \mathrm{i}\left(\lambda, \mathrm{p}_{-} \mathrm{i}, \gamma_{-} \mathrm{i}\right), \lambda=\left(\lambda_{-} 1, \ldots, \lambda \_\mathrm{N}\right)\right\}$, if $: \forall\left(\mathrm{i}, \lambda \_\mathrm{i}\right) \in(\mathrm{N}, \lambda)$

$$
U_{i}\left(\lambda_{1}{ }^{*}, \ldots, \lambda_{i}{ }^{*}, \ldots, \lambda_{N}{ }^{*}\right) \geq U_{i}\left(\lambda_{1}{ }^{*}, \ldots, \lambda_{i}, \ldots, \lambda_{N}{ }^{*}\right)
$$

Theorem1: A Nash equilibrium in terms of $\lambda$ for game $J=\left\{N, U_{i}\left(\lambda, p_{i}, \gamma_{i}\right), \lambda=\left(\lambda_{1}, \ldots, \lambda_{N}\right)\right\}$ exists and is unique.

Proof:

To prove existence, we note that each content's strategy space $\lambda_{i}$ is defined in the closed interval bounded by the minimum and maximum $\lambda$. Thus, the joint strategy space $\lambda$ is a nonempty, convex, and compact subset of the Euclidean space $\mathbb{R}^{\mathrm{N}}$. In addition, a Nash equilibrium exist if: the utility functions are concave with respect to $\lambda$ as can be seen from the second derivative test, in other words, A Nash equilibrium exists if:

$\forall \mathrm{i} \in \mathrm{N}$

$$
\frac{\partial^{2}}{\delta \lambda_{\mathrm{i}}^{2}} \mathrm{U}_{\mathrm{i}}\left(\lambda, \mathrm{p}_{i}, \gamma_{i}\right) \leq 0
$$

We have:

$$
\begin{aligned}
& \frac{\partial^{2}}{\delta \lambda_{\mathrm{i}}{ }^{2}} \mathrm{U}_{\mathrm{i}}\left(\lambda, \mathrm{p}_{i}, \gamma_{i}\right)=\frac{\partial^{2}}{\delta \lambda_{\mathrm{i}}{ }^{2}}\left(\mathrm{p}_{i} \times \mathrm{M} \frac{\lambda_{\mathrm{i}}}{\sum_{\mathrm{j}=1}^{\mathrm{N}} \lambda_{\mathrm{j}}}-\gamma_{i} \times \mathrm{M}\left(\lambda_{\mathrm{i}}-\phi_{\mathrm{i}}\right)\right) \\
& \frac{\partial^{2}}{\delta \lambda_{\mathrm{i}}{ }^{2}} \mathrm{U}_{\mathrm{i}}\left(\lambda, \mathrm{p}_{i}, \gamma_{i}\right)=-\frac{2 \mathrm{p}_{i} \mathrm{M}}{\left(\sum_{\mathrm{j}=1}^{\mathrm{N}} \lambda_{\mathrm{j}}\right)^{2}}+\frac{2 \mathrm{p}_{i} \mathrm{M} \lambda_{\mathrm{i}}}{\left(\sum_{\mathrm{j}=1}^{\mathrm{N}} \lambda_{\mathrm{j}}\right)^{3}}
\end{aligned}
$$

After simplification, we got: 


$$
\frac{\partial^{2}}{\delta \lambda_{\mathrm{i}}{ }^{2}} \mathrm{U}_{\mathrm{i}}\left(\lambda, \mathrm{p}_{i}, \gamma_{i}\right)=\frac{2 \mathrm{p}_{i} \mathrm{M}\left(\lambda_{\mathrm{i}}-\left(\sum_{\mathrm{i}=1}^{\mathrm{N}} \lambda_{\mathrm{i}}\right)\right)}{\left(\sum_{\mathrm{i}=1}^{\mathrm{N}} \lambda_{\mathrm{i}}\right)^{3}} \leq 0 \forall \mathrm{i} \in \mathrm{N}
$$

where: $\lambda_{\mathrm{i}}<\sum_{\mathrm{i}=1}^{\mathrm{N}} \lambda_{\mathrm{i}}$, then $\frac{\partial^{2}}{\delta \lambda_{\mathrm{i}}{ }^{2}} \mathrm{U}_{\mathrm{i}}\left(\lambda, \mathrm{p}_{i}, \gamma_{i}\right) \leq 0$.

Which ensures existence of a Nash equilibrium.

In order to prove uniqueness of Nash equilibrium for this game, we proceeded as follows:

We have assumed that there are many equilibrium states, and each one is influenced by the initial state of the game, in other words, the equilibrium states depend on the initial values which takes the parameter $\lambda$ of the game.

Then, we calculated the equilibrium corresponding to 2 situations that have different values of the parameter $\lambda$, and for each situation, the corresponding curve is plotted.

The following figures illustrate the curves obtained:
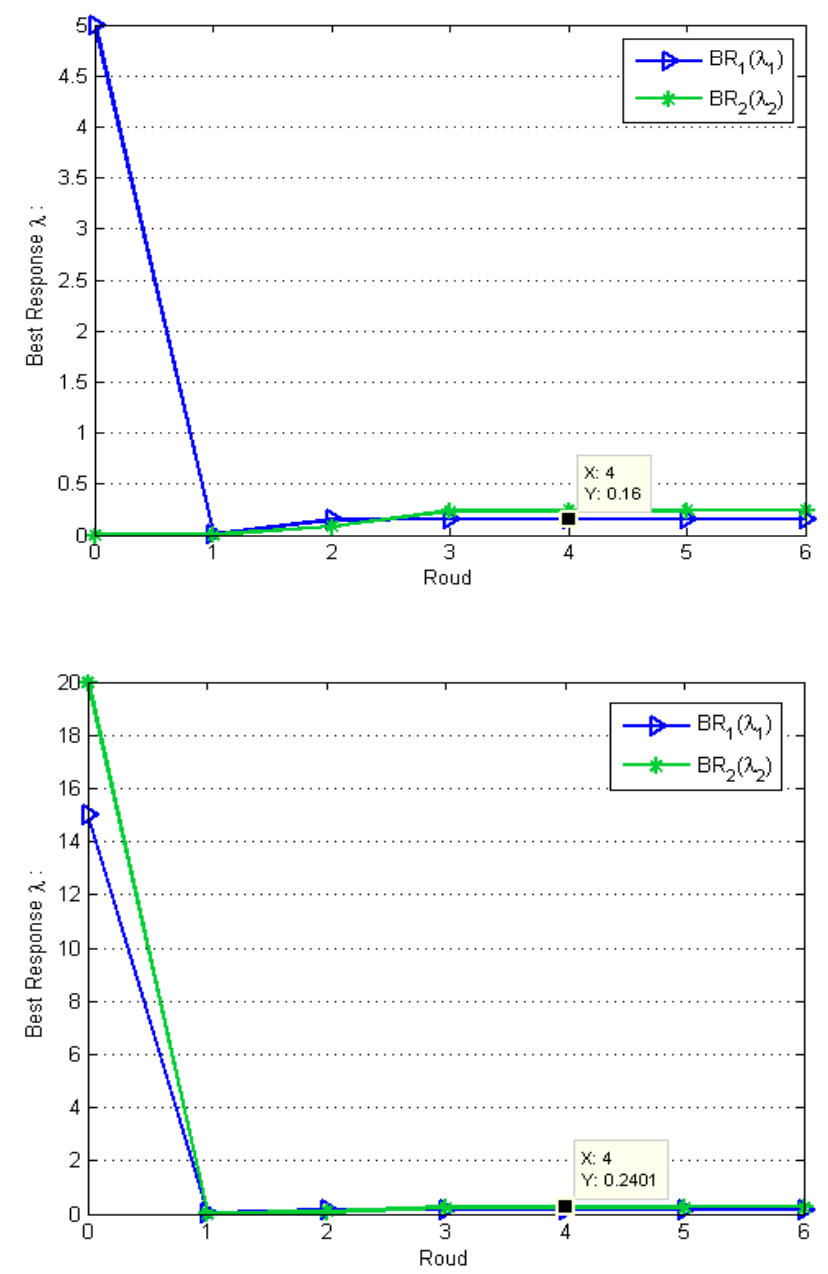

Figure 1. Nash Equilibrium for different values of $\lambda$

Figure 1 shows that, whatever the strategies selected by the players in the initial state, it always takes the same equilibrium state which is given by: $\lambda_{1}{ }^{*}=0.16$ and $\lambda_{2}{ }^{*}=0.24$.

These results ensure the uniqueness of Nash equilibrium

\subsection{Algorithm: Best Response Dynamics}

In this section, we study a fully distributed algorithm to learn the parameter equilibrium. Assuming that contents are selfish and choose dynamically each one the best lambda that maximize his profiles, the distributed algorithms can be thought of as protocols that players are programmed to follow. The design and 
analysis of distributed algorithms converging to equilibria in the context of games has also received considerable attention, most commonly convergence of best response dynamics.

Solutions of equations induces by vanishing the partial derivatives correspond respectively to the best response in terms of lambda $\mathrm{BR}_{\lambda}{ }^{\mathrm{i}}($.$) , of each content as a function of the strategies of its opponents.$ Since Nash equilibrium point is unique, then a best response-based dynamics would converge to the joint Lambda NE. The parameter best response dynamic is detailed in Algorithm 1.

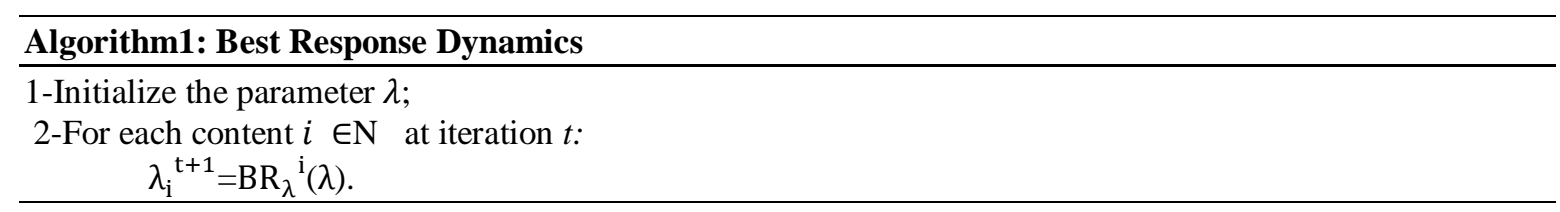

\subsection{Price of Anarchy}

The concept of social welfare [5] or total surplus [6], is defined as the sum of the utilities of all agents in the systems. It is well known in game theory that agent selfishness, such as in a Nash equilibrium, does not lead in general to a socially efficient situation. As a measure of the loss of efficiency due to the divergence of user interests, we use the Price of Anarchy (PoA) [7], this latter is a measure of the loss of efficiency due to actor's selfishness.

A PoA close to 1 indicates that the equilibrium is approximately socially optimal, and thus the consequences of selfish behavior are relatively benevolent. The term Price of Anarchy was first used by Koutsoupias and Papadimitriou [7] but the idea of measuring inefficiency of equilibrium is older.

As in [8], we measure the loss of efficiency due to actor's selfishness as the quotient between the social welfare obtained at the Nash equilibrium and the maximum value of the social welfare:

$$
\operatorname{PoA}=\frac{\sum_{\mathrm{i}=1}^{\mathrm{N}} \mathrm{U}_{\mathrm{i}}\left(\lambda^{*}\right)}{\max _{\lambda} \sum_{\mathrm{i}=1}^{\mathrm{N}} \mathrm{U}_{\mathrm{i}}(\lambda)}
$$

where:

$\sum_{\mathrm{i}=1}^{\mathrm{N}} \mathrm{U}_{\mathrm{i}}\left(\lambda^{*}\right)$ Represents the sum of utilities of all players in the Nash equilibrium. and $\sum_{\mathrm{i}=1}^{\mathrm{N}} \mathrm{U}_{\mathrm{i}}(\lambda)$ Represents the social welfare.

\section{NUMERICAL INVESTIGATIONS}

To test the validity of our theoretical study, we perform a numerical study based on the best response dynamics and the contents utility functions as well as the price of the anarchy. Therefore, we consider a system with two content $\mathrm{N}=2$ seeking to maximize their revenues, then, we plot curves to the Nash equilibrium, varying the parameters of the utility function. Table 1 summarizes the system parameter values used in this numerical study:

Table 1. System Parameters used for Numerical Examples

\begin{tabular}{cccccc}
\hline$P_{1}$ & $P_{2}$ & $\gamma_{1}$ & $\gamma_{2}$ & $\lambda_{1}$ & $\lambda_{2}$ \\
\hline 10 & 5 & 15 & 5 & 0.01 & 0.001 \\
$\gamma_{1}=\gamma_{2}$ & $P_{1}=P_{2}$ & $\phi_{1}=\phi_{2}$ & $\mathrm{M}$ & $\overline{\lambda_{1}}=\overline{\lambda_{2}}$ \\
5 & 15 & 5 & 3000 & 20 \\
$\underline{\lambda}_{1}=\lambda_{2}$ & $\overline{\mathrm{P}_{1}}=\overline{\mathrm{P}_{2}}$ & $\underline{\mathrm{P}_{1}}=\mathrm{P}_{2}$ & $\overline{\gamma_{1}}=\overline{\gamma_{2}}$ & $\underline{\gamma_{1}}=\underline{\gamma_{2}}$ \\
0 & 100 & 1 & 100 & $\underline{\gamma}_{1}$ \\
\hline
\end{tabular}

Figure 2 present curve of the convergence to Nash Equilibrium of the parameter $\lambda$. It is clear that the best response dynamics converges to the unique Nash equilibrium for the parameter $\lambda$.

We also observe that the speed of convergence is relatively high (4 iterations are sufficient to converge the Nash equilibrium of the parameter $\lambda$ ). 


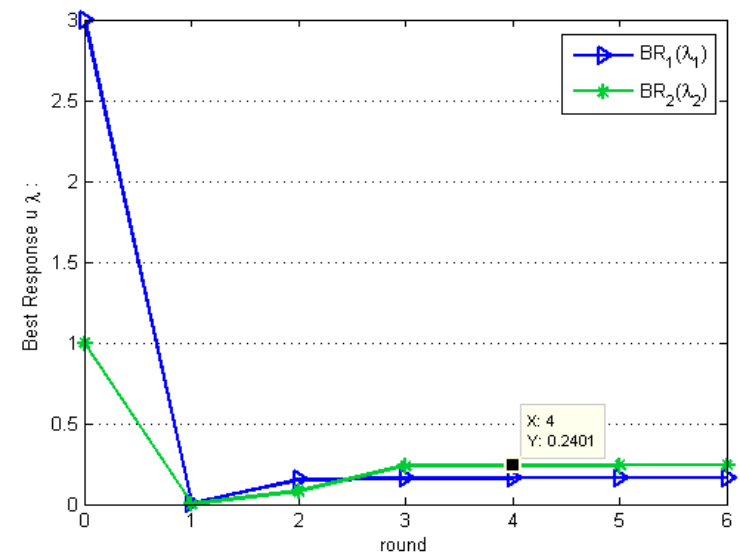

Figure 2. Convergence to the $\lambda$ Nash Equilibrium

Then, we plot in figures 3 and 4, respectively, the interplay of cost $\gamma$ and price $p$ on the parameter $\lambda$ at the Nash equilibrium

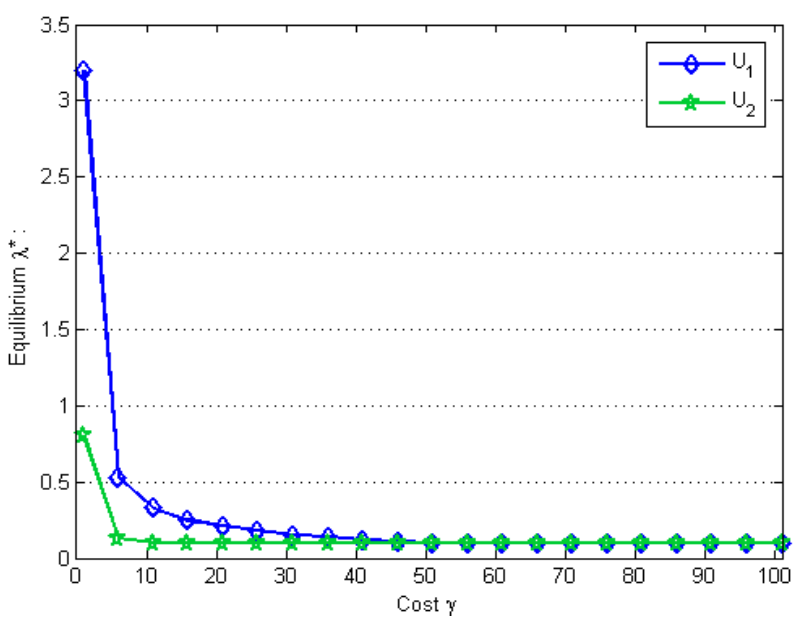

Figure 3: The interplay of cost $\gamma$ on $\lambda$

Figure 3 shows the cost impact on the parameter $\lambda$ : when the cost increases, $\lambda$ decreases, and then the time for a content $\mathrm{n}$ reaches a destination $\mathrm{m}$ increases progressively.

This means that users of this network will misjudge the content, which influence the revenue of content. Then, players are supposed to adopt situations which the parameter $\lambda$ is great, and whose production costs are slight to increase their profits. 


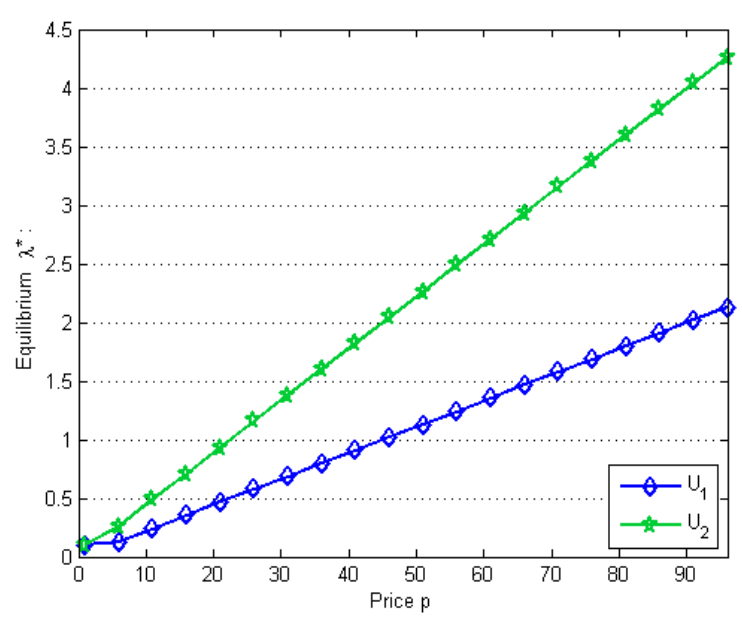

Figure 4: The interplay of price $\mathrm{p}$ on $\lambda$

Figure 4 shows the price impact on the parameter $\lambda$ : when the price increases, $\lambda$ increases, and the propagation time $t$ decreases, while subscribers of this network are satisfied, consequently, these contents will attract more customers, and this will increase their revenues.

In the following, we plot the variation of utility based on the system parameters.

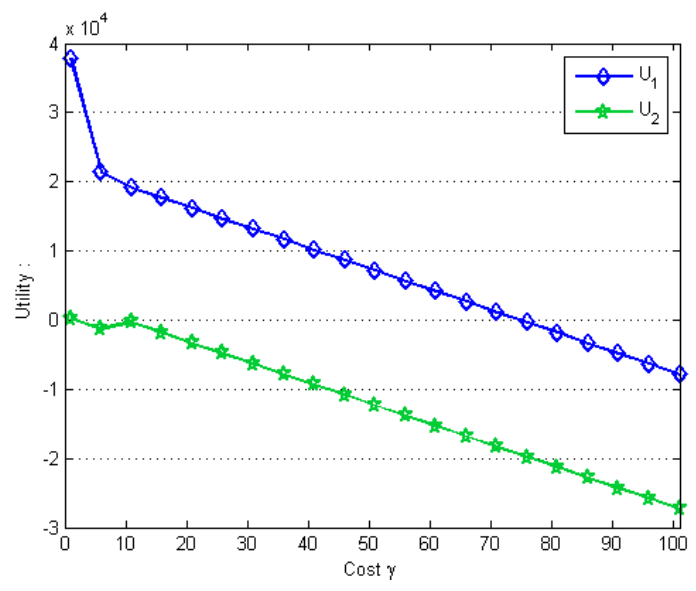

Figure 5. Variation utility as a function of the cost

Figure 5 shows that the utility reduced when the cost $\gamma$ get a high values, in this situation, players must raise their prices to increase their profits. 


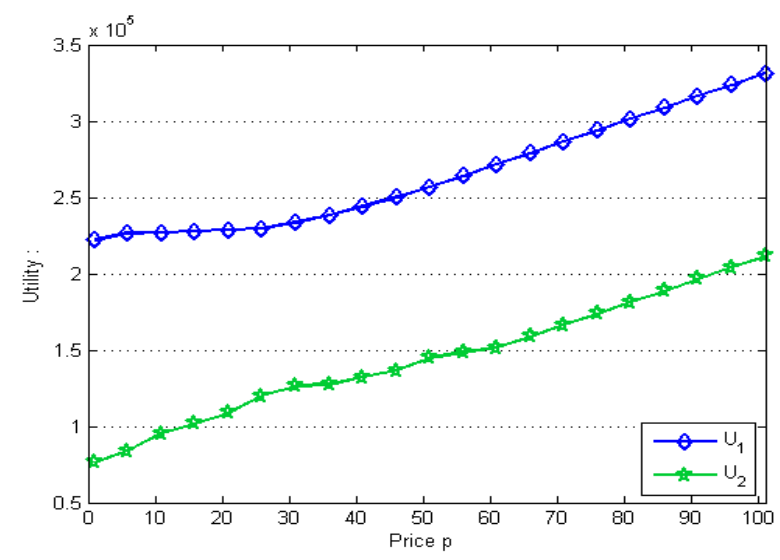

Figure 6. Variation utility as a function of the price $\mathrm{p}$

Figure 6 shows that the utility increases progressively when prices are increasing, this reveals that growing price ensures optimal revenues.

Finally, in this section, we show the impact of the system parameters on the system efficiency in terms of Price of anarchy:

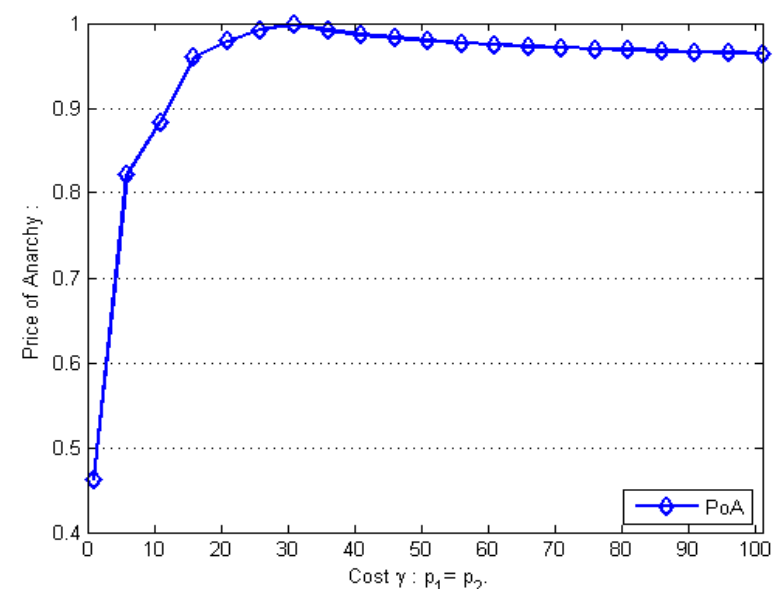

Figure 7. PoA variation as a function of the cost $\gamma$

Figure 7 shows the PoA variation as a function of cost $\gamma$ with fixed price $P_{1}=P_{2}$. For small values of $\gamma$, players are selfish $(\operatorname{PoA}<0.5)$, well each player tries to maximize his outcomes without considering the other player; for higher costs, the contents converge to the same price of anarchy which is close to 1, showing that players have cooperated for an optimum social welfare. We remark that the Nash equilibrium performs well and the loss of efficiency is only about $1 \%$. This result indicates that the Nash equilibrium of this game is good and socially efficient.

Figure 8 shows the PoA variation as a function of price $\mathrm{p}$ with fixed cost $\gamma_{1}=\gamma_{2}$. We remark that the price of anarchy reduce when the price rises. For the little price, the price of anarchy is to 1 which means, that players cooperate and the Nash equilibrium is socially efficient; while for higher prices, players have become more selfish, everyone reacts alone seeking maximize his outcomes. 


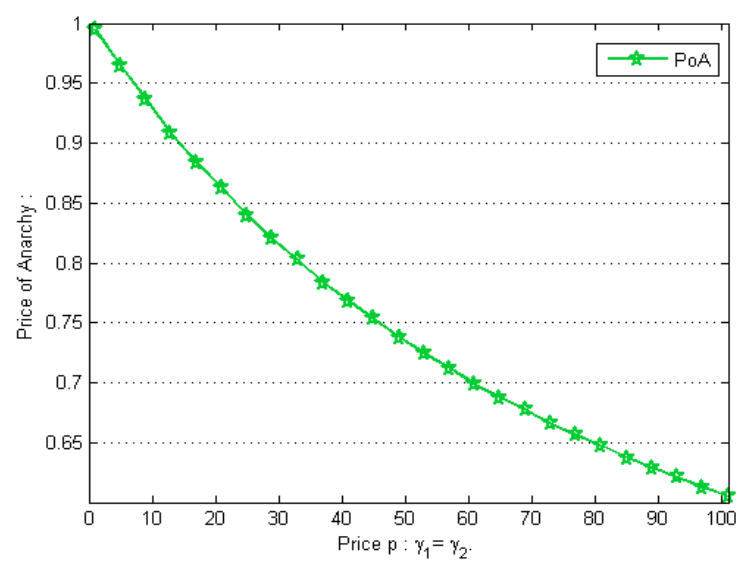

Figure 8. PoA variation as a function of the price

\section{CONCLUSION}

We have presented in this paper a type of competitive interactions between contents in a social network. There is a competition over a limited common set of destinations. We analyzed the behavior of the contents that seek to attract more consultations. The proposed model is based on a simple utility functions which takes into account not only the characteristics of a current content, but also of all other contents, also, it is based on two parameters describing each content price and cost of production.

We showed that the equilibrium becomes more important (socially efficient) when the cost of production of content is included in a given interval. We also showed that when the prices becomes higher, the competition becomes fierce.

\section{REFERENCES}

[1] Eitan Altman, "A Semi-Dynamic Model for Competition Over Popularity and Over Advertisement Space in Social Networks", INRIA Sophia-Antipolis, 2004 Route des Lucioles, 06902 Sophia-Antipolis Cedex, France.

[2] Eitan Altman, Parmod Kumar, Srinivasan Venkatramanan, Anurag Kumar, "Competition Over Timeline in Social Networks", INRIA Sophia-Antipolis, 2004 Route des Lucioles, 06902 Sophia-Antipolis Cedex, France.

[3] Eitan Altman, "Stochastic Game Approach for Competition Over Popularity in Social Networks", INRIA SophiaAntipolis, 2004 Route des Lucioles, 06902 Sophia-Antipolis Cedex, France.

[4] Rosen J., "Existence and Uniqueness of Equilibrium Points for Concave n-person Games, Econometrica 33: 520534, 1965.

[5] Maille, P. \& Tuffin, B., "Analysis of Price Competition in a Slotted Resource Allocation Game", in Proc. of IEEE INFOCOM. 2008.

[6] Varian, H., "Microeconomic Analysis", Norton New York. 1992.

[7] Papadimitriou, K. \& Koutsoupias, E. "Worst-Case Equilibria”, in STACS, pp.404-413. 1999.

[8] Guijarro, L., Pla, V., Vidal, J. \& Martinez-Bauset, J. "Analysis of Price Competition under Peering and Transit Agreements in Internet Service Provision to peer-to-peer Users", IEEE Consumer Communications and Networking Conference (CCNC2011), Las Vegas, Nevada USA, pp. 9-12. 2011. 